\title{
Adapting Inventory Models for handling various Payment Structures using Net Present Value Equivalence Analysis
}

September 27, 2013

Patrick Beullens (1), Gerrit K. Janssens (2)

(1) Mathematical Sciences and Southampton Management School, University of Southampton, SO17 1BJ, United Kingdom, P.Beullens@soton.ac.uk, Tel +44 238059 8884, corresponding author;

(2) Research Group Logistics, Hasselt University, B-3590 Diepenbeek, Belgium, gerrit.janssens@uhasselt.be

Accepted for publication in the International Journal of Production Economics on 22 September, 2013.

DOI Information:10.1016/j.ijpe.2013.09.013 


\begin{abstract}
Classic inventory models use average cost functions. It is generally accepted that these models should account for the time value of money. They do so not by considering the timing of cash-flows, but by including opportunity costs. The Net Present Value (NPV) framework has long been used to compare these models with. We formalise NPV Equivalence Analysis (NPVEA) under various payment structures, and apply it to a few classic inventory models. While taking the linear approximation is typically part of the process to find equivalence, the essence is to disregard the parameters of a classic inventory model but instead start from cash-flow structures between firms. It is demonstrated how this leads to different plausible interpretations of, or variations to, classic inventory models, in particular for payment structures that differ from conventional assumptions. We identify situations with negative holding costs, which indicates that more features from the real world must be added into the decision model. We illustrate that in addition to capital costs, firms can enjoy capital rewards. These rewards may not always affect the firm's inventory decisions, but are in general useful for finding the impact of changes to various parameters on the firm's future profits.
\end{abstract}

Keywords: deterministic inventory theory; net present value; payment structure; economic order quantity; economic production quantity; philosophy of modelling 


\section{Introduction}

The foundation that inventory theory is to account for the time value of money goes back to Harris (1913), who was careful to explain that his Economic Order Quantity (EOQ) model is largely about the trade-off with the opportunity cost of capital. His is the archetypal model of classic inventory theory, in which one minimises the average costs per unit of time. The holding cost is commonly found from the integration over a relevant cycle time $T$ :

$$
\frac{1}{T} \int_{0}^{T} h(t) I(t) d t
$$

where $I(t)$ is the inventory level at time $t$, and $h(t)=h$ the unit holding cost, typically taken to be time-invariant. Costs are not discounted according to their time of occurrence, but the time value of money is implicitly modelled by the inclusion into $h$ of the financial opportunity cost from stock investment. Typically, $h$ is taken to be of the form (Silver et al., 1998):

$$
h=\alpha v+f,
$$

where $v$ is the money invested per unit of product held in stock, $f$ the unit 'out-of-pocket' holding cost, and $\alpha$ the firm's continuous capital rate. A value $\alpha=0.20$ (time measured in years) is often used, and the putative view is that the financial holding cost dominates: $\alpha v>>f$.

The opportunity cost is also the foundation for the Net Present Value (NPV), which quite generally can be viewed to be the Laplace transform of a cash-flow function $a(t)$ in wich the Laplace frequency is taken to be $\alpha$ (Grubbström, 1967):

$$
\int_{0}^{\infty} a(t) e^{-\alpha t} d t
$$

As the time value of money is modelled explicitly, it would be incorrect to include into $a(t)$ the financial holding cost as used in classic models. Instead, it is retrieved in the linearised Annuity Stream (AS) function (Grubbström, 1980). The AS is the constant payment stream having the same NPV as a given stream of payments; for an infinite horizon, $\mathrm{AS}=\alpha \mathrm{NPV}$.

The comparison with NPV has been used at least as early as Hadley and Whitin (1963) and Hadley (1964), who demonstrate that Harris' model retains the lot-size relevant terms of the linear 
AS function. Grubbström $(1980,2007)$ shows how capital costs can be determined for inventories and work-in-process at several stages in more complex systems of production and inventory. See also Gurnani (1983). Porteus (1985) clarifies how the timing of expenditures and revenues relative to the cycle time of a regenerative process affects the valuation of capital costs, and illustrates using Harris' EOQ model. Teunter et al. (2000), Van der Laan and Teunter (2002), Teunter and van der Laan (2002), and Çorbacioğlu and van der Laan (2007) use the linear AS function for setting the unit holding costs at the different stages in systems of remanufacturing. They demonstrate that the mapping of the classic parameters to the AS function is not necessarily injective. Beullens and Janssens (2011) introduce the Anchor Point (AP) in NPV models, and show that its position in the supply chain can affect the valuation of capital costs at the different stages in the system.

In this article we use NPV to study the impact of payment structures on the unit holding cost and other parameters in classic models. Grubbström (1980) was perhaps first to introduce the term. Most studies that use NPV to retrieve the capital costs, except for Porteus (1985) and Beullens et al. (2013), adopt 'conventional' assumptions. Loosely speaking, this means that costs or revenues are assumed to occur either the moment that some (physical) process in the system initiates or terminates, such as the transfer of a batch of materials, or continuously at the rate of process transformation, such as a production rate. As the timing of payments is not described in classic inventory models, this first guess often leads to satisfactory results. However, trends in inventory management, including the use of consignment stocks and credit delays, show that a wider variety of payment structures are adopted in practise. The question is then under which variety of payment structures the classic models, and their solutions, can still be used, or how they should be adapted.

The study of equivalence is formalised as NPVEA in Section 2, and a basic idea from propositional logic introduced. We belief that this makes it easier to present results, and their logical consequences, succintly. In Section 3 payment structures are defined and common examples presented. In Section 4 to Section 7, the approach is applied to a few well-known inventory models. Next to finding out the strengths and weaknesses of these models, it leads to a number of simple variations which do not appear in our literature, but should be of relevance in the context of various practical applications. As a general conclusion, we find that the study of equivalence under various payment structures can be of great help to better understand inventory theory, and increase and extend its applicability.

The article considers infinite horizon models with constant demand, but NPV can also be used for dynamic lot-sizing, see e.g. Grubbström (2013). NPV is not the only possible framework to help 
making finanical decisions about the future, see e.g. Xu et al. (2012) for a comparison with real options.

\section{NPV Equivalence Analysis}

A satisfactory understanding of a classic inventory model is not automatically arrived at when adopting the NPV viewpoint, as classic inventory theory is insufficient to describe cash-flow functions. The extra degrees of freedom in NPV imply that there is an, in principle infinite, number of possible interpretations of a classic model. Insight into the meaning of a classic model's (cost) parameters is best achieved by not using them in the NPV reference model. Application of NPVEA leads to an enriched interpretation of classic inventory theory, and further development.

\subsection{Equivalence Framework}

A firm is involved in some activity A. The activities in inventory theory are typically about the movement, transformation, and stockage of products or services. A firm has to deal with flows of goods and services inside its boundaries as well as those exchanged with the outside world. The latter may include suppliers, third parties, employees of the firm, providers of equipment and other supplies, and customers. Let $X$ denote a scenario by which all these flows of goods and services, needed to perform $\mathrm{A}$ in the time interval $[0, \infty)$, are organised in a certain manner. Call $\mathcal{A}$ the set of all such scenarios available to the firm.

Using classic inventory theory, one constructs an average profit function $P(X)$ for the firm related to A that reflects both real expenses and revenues, and capital costs as a function of $X$. The problem calls for finding $X^{*}=\arg \max _{X \in \mathcal{A}} P(X)$. For A to be worthwhile, $P\left(X^{*}\right)>0$. For constant revenues, one can alternatively minimise a cost function $C(X)$. In the NPV framework, one starts from the cash-flows related to A that the firm exchanges with the outside world. The cash-flow function of interest, $a(X)$, describes the size and timing of these in- and outgoing cash-flows as a function of $X$.

The problem calls for finding $X^{*}=\arg \max _{X \in \mathcal{A}} \int_{0}^{\infty} a(X) e^{-\alpha t} d t$. The NPV (or AS) related to $X^{*}$ should be non-negative to justify engagement in A.

The question of equivalence deals with the problem of establishing whether, and if so, under which conditions, the two frameworks can find the same scenario $X^{*}$ to be optimal. It is now well-known that 
it is impossible to achieve in but trivial and non-interesting models ${ }^{1}$. Better results are known to be obtainable when comparing with a linear approximation of the NPV (AS) function. Notwithstanding the worth of unapproximated or higher order NPV models, see e.g. Disney and Warburton (2012), the study of linear approximations remains useful for the following reasons. First, these models often offer analytical solutions and insight. Second, they are typically accurate if we account the fact that managers commonly do not wish to implement solutions with very long cycle times. Third, at least since Hadley and Whitin (1963) classic inventory theory is thought to represent the linear approximation of NPV fairly accurately. Within the range of practical cycle times discussed above, it should be an accurate theory if it is indeed a theory about the linear approximation. NPVEA shows that this is in general not the case: the linear approximation is needed in proving which first order effects are responsible for the difference. This opens the route to improving the theory.

We adopt from the literature the notion of NPV models being more accurate in reference to a particular 'real-world' situation. Because a classic model is based on less information, it might be (very close to being) the linear approximation of potentially many NPV models. Equivalence to a reference model reveals applicability of the classic model to that situation, and the conditions under which equivalence holds help to complete this interpretation by specifying how to set the classic model's parameters. There are many possible reference models and hence possible interpretations.

It is formalised as follows. Let $q$ be a propositional variable about the applicability of a particular classic inventory model. We use $q$ to express in shorthand the belief that this model can be used. Let $p$ be a propositional variable about the validity of a particular cash-flow function $a(X)$. Hence, $p$ expresses in shorthand which NPV model is the reference. Two types of results are obtainable:

1. $p \rightarrow \neg q$, and $q$, infer $\neg p$ by modus ponens;

2. $p \rightarrow q$.

A result of the type $p \rightarrow \neg q$, which we call 'non-equivalence', implies the following:

- the applicability of the classic model cannot hold under $p$;

- an interpretation of the classic model is not obtainable from $p$;

\footnotetext{
${ }^{1}$ This follows from the fact that linear models cannot account for the cumulative impact of interests on interests, see the discussion in Haneveld and Teunter (1998).
} 
- corrections to the classic model may be found such that its new version $q^{\prime}$ may give $p \rightarrow q^{\prime}$ (repairable equivalence).

A result of type $p \rightarrow q$, which we call 'equivalence', provides the following information:

- the classic model is applicable under $p$;

- an interpretation of the classic model follows from $p$; the parameters of the classic model can be made explicit functions of the payment structures or other features of $p$.

Let $\mathcal{P}$ be the universe of all cash-flow functions $a(X), \forall X \in \mathcal{A}$. As typically $p \neq \mathcal{P}$, a result of any of the two types obtained for $p$ does hence not exclude that $\exists p^{\prime} \in \mathcal{P}, p^{\prime} \neq p$ such that either

$p^{\prime} \rightarrow \neg q$ or $p^{\prime} \rightarrow q$. As long as no result for $p=\mathcal{P}$ is obtained, the search for equivalence and valid interpretations of classic models is incomplete. This should be taken into account for evaluating the results presented in further sections.

\subsection{Choosing the reference model and interpretation of results}

The reference model is based on arbitrary choice. Indeed, the 'Modus Operandi' (MO) of NPVEA is as follows: Take an existing (inventory) model $q$ not based on cash-flow functions. Identify the logistics process in this model. Make up a story of how a set of firms involved in making this process work exchange cash-flows with each other and their outside world. Instead of using the (cost) parameters of $q$, make assumptions as to specify each firm's cash-flow function. With the model $p$ so constructed, perform the equivalence analysis, where you keep track of the conditions or additional assumptions you are making in the process of finding (repairable) equivalence.

The following two arguments cannot in principle discredit the choice of $p$. The first is demanding that reference models are to reflect the real world. This is not always desirable as a novel $p$ might, for some firms, produce better results relative to current practise. The second is demanding that $p$ should adopt all and only those assumptions used by the designers of $q$. Because of the information gap, this is impossible, and we must look beyond the original intentions of the designers of $q$ by making reasonable guesses about e.g. the payment structures.

What justifies the choice of a particular $p$ is the equivalence result itself. This is no different in spirit to what is called in Deutsch (2011) the scientific method of discovery through conjecture and verification. While any $p$ has the potential of providing a (novel) interpretation of $q$, this remains 
a conjecture until after an equivalence analysis has found $p \rightarrow q$ or $p \rightarrow q^{\prime}$. A model $p$ which does not lead to either of these two results, and hence produces $p \rightarrow \neg q$ that remains unexplained, is of less scientific interest unless this result would be on itself surprising. The choice about $p$ is hence not completely arbitrary as there are many more models to construct leading to this type of result only, and this does not help us in identifying which bits of $q$ (and its underlying theory) or worth keeping. However, if a theory can be developed as how to fix $q$ to become $q^{\prime}$ such that $p \rightarrow \neg q$ leads to $p \rightarrow q^{\prime}$, then this is of scientific interest, which justifies $p$ in its role as reference model to this $q$.

A result of type $p \rightarrow q$ is of interest as it offers sufficient details about assumptions that need to be made as to arrive at a good understanding of how to apply the classic inventory model to the reference situation. That is why it is important when building the reference model to discard those (cost) parameters of the classic model of which one wishes to find an interpretation. See Example 7 in Section 4.2 for an example. In any case, the unit holding cost $h$ cannot be used for reasons explained in Section 1. If equivalence is found with respect to many reference models, a rich understanding of the model's applicability and range of valid interpretations is obtained. A result $p \rightarrow \neg q$ implies that a situation is identified in which $q$ cannot apply. It does not imply that $q$ or its underlying theory contains flaws in any absolute sense, but only relative to $p$. There may be other situations (i.e. other reference models) in which there $i s$ equivalence. Non-equivalence may provide the starting point to the development of new theory that explains why one should, in the future, construct $q^{\prime}$ rather than $q$ such that instead of $p \rightarrow \neg q$ one finds $p \rightarrow q^{\prime}$. Concepts derived from NPVEA that help achieving this include the Anchor Point (AP) and the Supplier's Reward (SR), see Beullens and Janssens (2011).

\section{Payment Structures}

The accurate specification of $a(t)$ is important. A cash-flow function can contain discrete finite payments at particular moments in time as well as payment streams over time intervals. Most financial transactions in the real world are arguably of the former type. The latter are mathematically convenient to model a stream of discrete payments occurring in quick succession. 


\subsection{Payment structure, symmetry, and timing}

A financial transaction between a sender and a receiver is assumed to be triggered by an event occuring at some future moment in time, say $t(t \geq 0)$, called the event time. In the systems under study, the event can be linked to activities at one of these parties, such as the start or completion of an operation of movement, transformation, or stockage. The event is assumed to determine the size of payment, say $w(w \geq 0)$, called the event payment.

Definition 1. Payment Structure. The Payment Structure (PS) between a sender and a receiver in an (NPV) decision model specifies at what future times, relative to the event time $t$, which amount of the event payment $w$ is paid out by the sender, and at what future times, relative to $t$, which amount of $w$ arrives at the receiver.

Example 1. Consider the transfer of a batch of $Q$ products from a supplier to a buyer, and let this batch arrive at the buyer at event time $t$. Let $v Q$ be the event payment. The PS then expresses when, relative to $t$, which amount of $v Q$ is leaving the buyer, and when, relative to $t$, which amount of $v Q$ is arriving at the supplier.

It is clear that the number of possible PSs is only limited by our imagination. We focus here on a few simple and representative cases.

Definition 2. Payment Symmetry. If the sender pays out an amount $w^{\prime}$ at time $t^{\prime}$ and the receiver gets that amount $w^{\prime}$ at time $t^{\prime}$, the payment is called symmetric.

A payment that is not symmetric is called asymmetric. It occurs when e.g. the receiver incurs a $\operatorname{cost} c^{\prime}>0$, or when $w^{\prime}$ arrives at the receiver with some delay $d^{\prime}>0$ at time $t^{\prime}+d^{\prime}$. Figure 1 illustrates two symmetric payments of $a_{1}$ and $a_{2}$, and one asymmetric payment of $a_{3}$. If payment symmetry holds for all cash-flows between all parties in an (inventory) model, we say that the Assumption of Symmetry (AoS) is satisfied ${ }^{2}$. Adopting the AoS simplifies the PS definition as we only need to specify it for one of the two parties. The name 'symmetry' is enforcing the idea that the other party's cash-flow of this payment is the negative of the first. Symmetry does not imply that payment has to occur at the event time $t$ or that the amount has to equal the event payment $w$. In this article we assume, except for the example in Figure 1, symmetrical payments throughout.

\footnotetext{
${ }^{2}$ It corresponds to Axiom 1 in the framework of Beullens et al. (2013).
} 


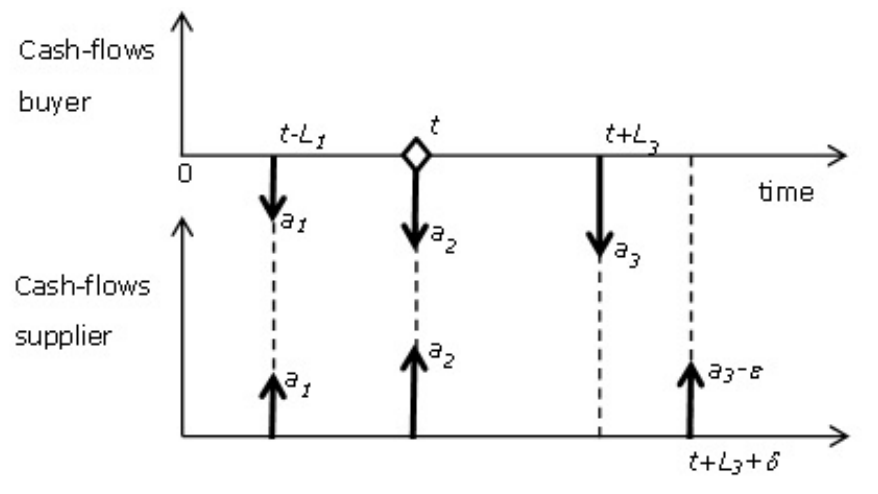

Figure 1: An event at $t$ triggers symmetric payments $a_{1}$ (CIA) and $a_{2}(\mathrm{C})$ and asymmetric payment $a_{3}(\mathrm{CR})$ with cost $\varepsilon$ and delay $\delta$ to the receiver

Definition 3. Payment Timing. The sender pays out $w^{\prime}$ at $t^{\prime}$. If $t^{\prime}=t$, the payment is Conventional (C); if $t^{\prime}>t$, it is a CRedit (CR) payment; if $t^{\prime}<t$ it is a Cash-In-Advance (CIA) payment. If in addition $w^{\prime}=w$ and the payment is symmetric, it is a conventional, credit, and cash-in-advance payment structure, respectively.

The three payment types are illustrated in Figure 1 by $a_{1}, a_{2}$, and $a_{3}$, respectively, where $t$ is the event time in each case. CR and CIA can come in several 'flavours' depending on modelling details (see also Section 4), and more complex PSs arise when different parts of $w$ have to be paid for according to different timings (imagine, for example, $w=a_{1}+a_{2}+a_{3}$ in Figure 1). We also say that $\mathrm{C}$ payments have zero delay, CR payments a strictly positive delay, and CIA payments a strictly negative delay.

Under the C PS, the sender pays out the event payment at the event time. Payment is 'immediate' and 'in full.' It is found in most of the work using NPV for the study of systems of production and inventory. Applied to Example 1, the cash-flow function for the buyer is the impluse of height $-v Q$ at $t$, and the supplier's cash-flow function is the negative of this: $+v Q$ at $t$. Let $\alpha_{b}$ and $\alpha_{s}$ be the capital rates for buyer and supplier, respectively, then the NPV values are $-v Q e^{-\alpha_{b} t}$ and $+v Q e^{-\alpha_{s} t}$. Under the CR PS, the sender pays the event payment some time after the event time. Applied to Example 1, if the buyer pays at time $t+L,(L \geq 0)$, its NPV is $-v Q e^{-\alpha_{b}(t+L)}$. It is found in the literature on trade credits, see e.g. Rachamadugu (1989), where typically $L$ is assumed to be a constant. Within this stream of literature, various extensions have been investigated. For example, Chung and Liao (2009) consider the case that the buyer has to pay according to C or CR, depending 
on the size of $Q$, whereas Teng et al. (2007) include the consideration of interest charges from the supplier. One can also identify CR in Porteus (1985), but he studies regenerative processes of cycle length $T$ and $L$ is a function of $T$. There is a fundamental difference between these two 'flavours' of credits with implications for equivalence analysis (see Section 4). Under the CIA PS, the sender pays the event payment prior to the event time. There is relatively little research into this in the inventory literature. If, for example, a payment is specified to take place at some time $t-L$, then it must hold of course than $t-L \geq 0$ for this PS to be applicable ${ }^{3}$. One can mix payment types and flavours to find new PSs. In Example 1, for example, the buyer may pay a deposit $x v Q(0 \leq x \leq 1)$ at a time $t-L(=\mathrm{CIA})$, when e.g. he places the order, and pays the remainder $(1-x) v Q$ at some time $t+L^{\prime}(=\mathrm{CR})$.

\subsection{Payment streams, and payments in the supply chain}

If many events occur consecutively in some time interval, it may be convenient to revert to payment streams, as in the following example.

Example 2. A firm satisfies demand for a product at a constant rate $y$ per unit of time in a time interval $[t, t+T]$, and is to receive $r$ per unit of product.

It can be given a continuous or discrete interpretation. The first represents and infinite number of events with infinitesimally small interarrival times (e.g. a flow of oil). The discrete interpretation is that of a finite number of $y T$ events occurring at equally spaced event times in $[t, t+T]$. When the interarrival time between events is sufficiently small, the continuous interpretation is a fair approximation. We refer to the collection of these (discrete) events as an event stream. A C PS in both cases means that the cash-flow received for the event at time $t^{\prime} \in[t, t+T]$ arrives at $t^{\prime}$; in Example 2 the event stream at constant rate $y$ over $[t, t+T]$ hence produces the payment stream at constant rate +ry over this interval, with NPV:

$$
\mathrm{NPV}(\mathrm{C})=\int_{t}^{t+T} r y e^{-\alpha x} d x=\frac{r y\left(1-e^{-\alpha T}\right) e^{-\alpha t}}{\alpha}
$$

One can interpret the discrete case as satisfying different customers. Asking from every customer cash-in-advance of duration $L$ then implies that $\mathrm{NPV}(\mathrm{CIA})=\mathrm{NPV}(\mathrm{C}) e^{\alpha L}$, and giving every customer a credit of duration $L$ that $\operatorname{NPV}(\mathrm{CR})=\mathrm{NPV}(\mathrm{C}) e^{-\alpha L}$. It may be equally assumed that all products

\footnotetext{
${ }^{3}$ Even general relativity theory does not allow for travelling back in time.
} 
are delivered to the same customer as part of e.g. a just-in-time delivery system - the above NPV values for C, CR, and CIA apply. Alternatively, it could be that the firm then receives the full payment ryT from the customer at some discrete moment, say $t$. This PS is a 'variable' type of CIA as each product receives a different negative delay relative to its event time $x$ in (4). While still delivering just-in-time, the NPV of the firm becomes $r y T e^{-\alpha t}$. Hence, while an event stream often results in a payment stream, it may also produce a discrete payment. Likewise, a discreet event may lead to a discrete payment or a payment stream, or some combination, as in Example 3 below.

Most firms in a supply chain have at least two external parties to consider, their supplier and their buyer, and thus at least two PSs in their cash-flow function, as in the following example.

Example 3. Let the firm of Example 2 be the buyer of Example 1. The buyer receives a batch of $Q$ products at time $t$, and uses $Q$ to satisfy demand at a constant rate $y$.

We need two PSs, one between supplier and buyer, and the other between the buyer and its customer(s). One solution is to adopt C PSs as developed in Examples 1 and 2. Here, we consider a few alternatives. The arrangement whereby the buyer receives the revenue under $\mathrm{C}$, and agrees with its supplier to pay $v$ for a product at the moment it is actually sold to a customer, is known in the literature as full consignment. The PS with the supplier is a 'variable' type of CR as relative to event time $t$ each product receives a different delay. The NPV for this combination of PSs is:

$$
\mathrm{NPV}=\frac{(r-v) y\left(1-e^{-\alpha \frac{Q}{y}}\right) e^{-\alpha t}}{\alpha} .
$$

A common variation is partial consignment, whereby the buyer pays $x v Q(0 \leq x \leq 1)$ at time $t(=\mathrm{C})$, where $x v$ may correspond to the supplier's unit cost, for example, but pays $(1-x) v$ for a product the moment it is actually sold $(=\mathrm{CR})$ :

$$
\mathrm{NPV}=\frac{(r-(1-x) v) y\left(1-e^{-\alpha \frac{Q}{y}}\right) e^{-\alpha t}}{\alpha}-x v Q e^{-\alpha t}
$$

There are many other variations possible by mixing payment structures. Example 4 illustrates how out-of-pocket holding costs are typically modelled.

Example 4. Consider the firm of Example 3, receiving the batch $Q$ at time $t$ to meet a constant demand rate $y$. The firm incurs a cost $f$ per unit of product for every unit of time it is held in inventory. 
The NPV of this under $\mathrm{C}$ is:

$$
\mathrm{NPV}(\mathrm{C})=\int_{0}^{T}-f y(T-x) e^{-\alpha(x+t)} d x=-f y\left[\frac{T}{\alpha}-\frac{1-e^{-\alpha T}}{\alpha^{2}}\right] e^{-\alpha t}
$$

This may in many circumstances be a reasonable model. However, sometimes the warehouserelated costs may be rather independent of the actual inventory levels at any point in time. For example, if the largest out-of-pocket cost is an insurance costs paid out as an annuity stream at the rate of the average inventory level, the NPV value is simply $-f y T / 2 \alpha$.

In conclusion, the examples above illustrate that the payment structures in the logistics process can greatly determine the NPV of the activity. We will, arguably, obtain a more accurate inventory management theory when taking payment structures more explicitly into account than what perhaps has prevailed in the existing literature.

\section{Harris' EOQ model}

In this and following sections, we apply NPVEA, using different PSs, to a few classic models. The EOQ model is well-known. See also Figure 2, top graph. A firm satisfies a constant demand rate $y$ without shortages. It procures, or produces at infinite rate, in batches of equal size $Q=y T$, where $T$ is the cycle time. With each batch, a set-up cost $s$ is incurred, and $h$ is the unit holding cost. The

value for $T$ that minimises $C(T)=s / T+h y T / 2$ is $T^{*}=\sqrt{2 s / y h}$, and $C^{*}=\sqrt{2 s h y}$. While necessary to assume $s y h>0$, it is sufficient and seems fair to say that the typical interpretation is that $s, y$ and $h$ are three independent parameters that each can take arbitrary but strictly positive values. We write $q(E O Q)$ to express the applicability of the class of model instances $\{C(T \mid s, y, h) \mid s, h, y>0, T>0\}$.

The NPV reference model is based on cash-flow functions. It is not derived from the cost parameters $s$ and $h$, but from payment structures with relevant parties, say a supplier, a customer, and a third party logistics provider. The third party transports the batches from the supplier and rents out warehouse space to the firm. Three types of events trigger payments: a batch of supplies arriving, sales occurring, and stock keeping. While the first type is discreet, the latter two are modelled as event streams at rate $y$ and at unity rate, respectively. In the PS with the supplier, the event time is the arrival of a batch, and the event payment is $-w Q$; in the PS with the customer, the event payment stream is at the rate +ry per unit of time; in the PS with the third party, the arrival of a 
batch triggers event payment $-k$ (independent of batch size), and stock keeping triggers the event payment stream at the rate of $-g$ per unit of time and unit of product in stock.

Various reference models are derived by specifying the payment timings differently. This results in different interpretations of Harris' model, as illustrated below.

\subsection{Conventional interpretation versus negative (unit) holding costs}

$\mathbf{p}(\mathbf{E O Q}, \mathbf{C})$. The first reference model adopts $\mathrm{C}$ for all PSs. See Figure 2, second graph from the top. While similar to Hadley and Whitin (1963); Hadley (1964), we present this model to illustrate the main components of NPVEA as well as to serve as the point of comparison for other reference models. The annuity stream (AS) profit function of the firm is given by:

$$
\Pi(T)=r y-\left(k+w y T+\int_{0}^{T} g e^{-\alpha t} y(T-t) d t\right) \sum_{i=0}^{\infty} \alpha e^{-i \alpha T},
$$

or, using (7), where $g=f$ :

$$
\Pi(T)=r y-(k+w y T) \frac{\alpha}{1-e^{-\alpha T}}-g y\left[\frac{T}{1-e^{-\alpha T}}-\frac{1}{\alpha}\right]
$$

Thus $p(E O Q, C)$ represents the validity of this cash-flow function.

We derive a linear approximation of the AS function by Maclaurin expansion of the exponential terms in $\alpha T$, and only retaining the terms linear in $\alpha$ :

$$
\bar{\Pi}(T)=(r-w) y-\frac{k}{T}-\alpha \frac{k}{2}-[\alpha w+g] \frac{y T}{2}-\alpha \frac{g}{6} T \frac{y T}{2} .
$$

Assuming $g<<\alpha w$, we can further drop the last term from $(10)$, and then $T^{*}=\sqrt{2 k /[y(\alpha w+g)]}$. We find $p(E O Q, C) \rightarrow q(E O Q)$ under the conditions: $s=k ; h=\alpha w+g ; g<<\alpha w ; \alpha T<<1$, and (2) is confirmed for $f=g$ and $v=w$. The EOQ model is fairly accurate for use in the reference situation under the putative view that financial holding costs dominate the out-of-pocket holding costs, whereas all these classic cost parameters receive a precise interpretation in terms of which cost or revenue cash-flows they represent, with which firms these are exchanged, and when.

Note that if Harris' square root formula finds a $T^{*}$ such that $\alpha T^{*}<<1$ is violated, then this result might be inaccurate since (10) is not valid across this range. Similar considerations will re-occur 
throughout the rest of this paper. In each case, good numerical solutions to the unapproximated NPV function such as (9) (or a higher order approximation of it) can be obtained, a function which is available from the reference model in NPVEA. However, if the linear approximation is an accurate representation when $\alpha T<<1$, if $\alpha \approx 0.2$, and if realistic values of $T$ cannot be much above 1 (recall the discussion of practical cycle times in Section 2.1), then the linear approximation and thus Harris' function is still a good representation of the decision problem as long as the model includes a constraint specifying e.g. that $T \leq 1$.
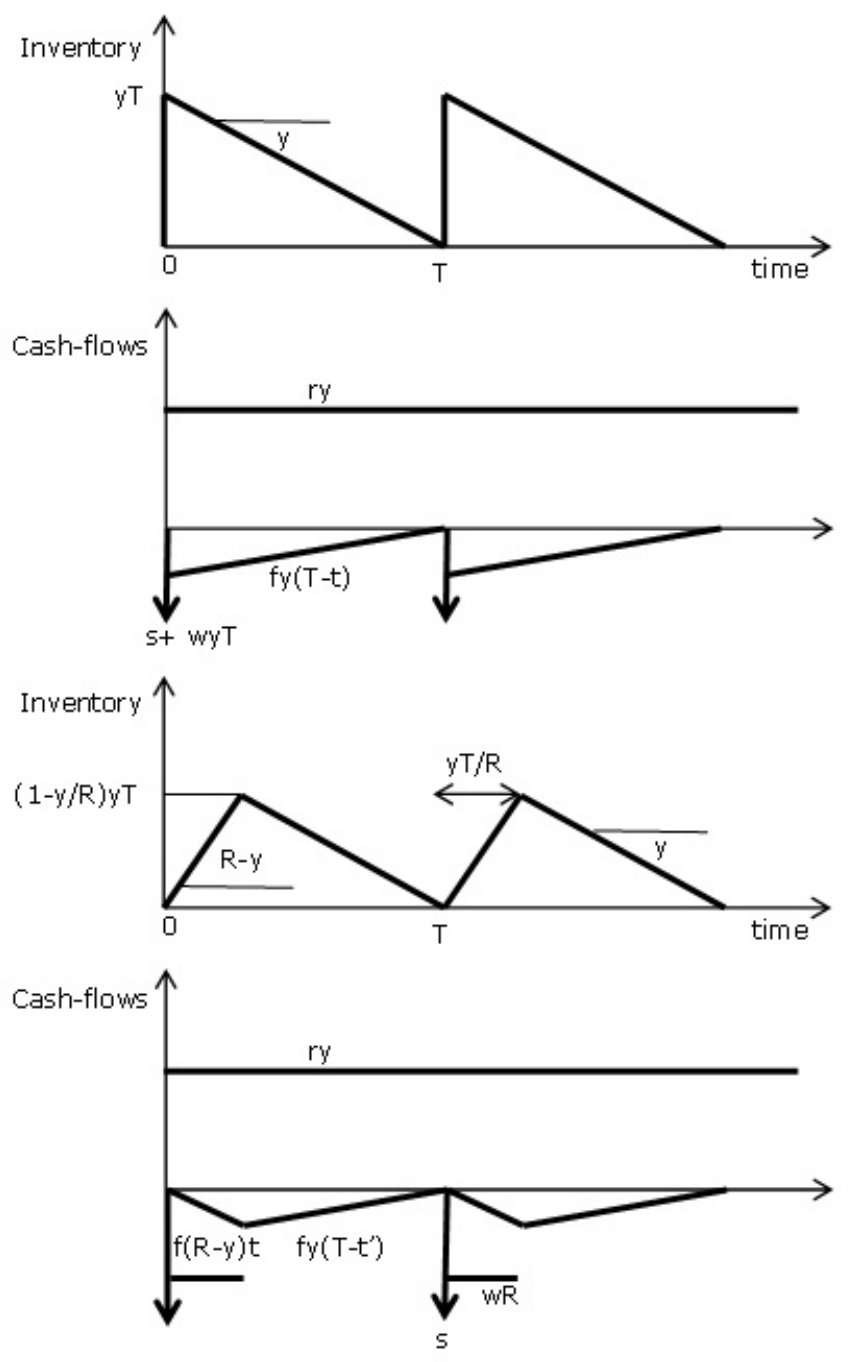

Figure 2: EOQ and EPQ under conventional payment structures

In the following reference models, we immediately take $s$ instead of $k$ and assume that $g=0$ for reasons of notational convenience but without loss of generality. 
$\mathbf{p}(\mathbf{E O Q}, \mathbf{C} / \mathbf{C R})$. Take the model $p(E O Q, C)$ but change the PS with the supplier, ceteris paribus, as follows. The supplier requires $x$ percent of $w$ to be paid under $\mathrm{C}$, at event time $t$ say, and the remainder under $\mathrm{CR}$ at time $t+L+z Q / y$, where $L$ is a given non-negative delay, and $z$ a given non-negative (fractional) number, both specified by the supplier. (The buyer chooses $Q$.) This payment structure expresses that the supplier is willing to give more credit to a buyer who, relative to his annual purchasing volume $y$, orders in larger lot-sizes. The rationale of this would be that when ordering in larger batches, the supplier receives his revenues earlier (an effect called the supplier's reward in Beullens and Janssens (2011) and described in detail in Beullens et al. (2013)) and is hence more supportive in giving such buyers a credit benefit in return. We can also say that the fixed delay $L$ is as used in the trade credit literature (Rachamadugu, 1989), and $z Q / y$ is a cycle-time variable credit as in Porteus (1985). The AS function is now:

$$
\Pi(T)=r y-\left(s+x w y T+(1-x) w y T e^{-\alpha(L+z T)}\right) \sum_{i=0}^{\infty} \alpha e^{-i \alpha T} .
$$

Maclaurin expansion of exponential terms with respect to $\alpha T$ and $\alpha z T$, and retaining only the terms linear in $\alpha$, gives the approximation:

$$
\begin{gathered}
\bar{\Pi}(T)=\left(r-x w-(1-x) w e^{-\alpha L}\right) y-\frac{s}{T}-\alpha \frac{s}{2} \\
-\alpha\left(x w+(1-x) w(1-2 z) e^{-\alpha L}\right) \frac{y T}{2} .
\end{gathered}
$$

Let:

$$
h=\alpha\left(x w+(1-x) w(1-2 z) e^{-\alpha L}\right) .
$$

Hence, the unit holding cost can become zero or negative. If $h>0$, then $T^{*}=\sqrt{2 s / y h}$, else ' $T^{*} \rightarrow \infty$ '. The latter may be inaccurate given the range in which (12) is an accurate approximation, but nevertheless it indicates that (if $h \leq 0$ ) there is no local optimum within the practical range of cycle times (say $T<1$, as previously discussed). Insight into the behaviour of the NPV for larger cycle times, and hence on the optimality of infinite cycle times, can only be assuredly obtained from the unapproximated function (11). Application of L'Hôspital's rule shows that $\lim _{T \rightarrow \infty} \Pi(T)=r y-s$ when $x=0$ for any value $z>0$, but $\lim _{T \rightarrow \infty} \Pi(T)=-\infty$ for $x>0$. The first limit shows when an infinite cycle time is optimal; for even the tiniest credit $z>0$, as long as nothing has to be paid at 
the event time, the firm would find it optimal to order all future demand at once. The approximation (12) identifies infinite cycle times as optimal only when $z \geq 1 / 2$ (for $x=0$ ), as in the other case the local optimum is found. The second limit shows that for even the tiniest value $x>0$, then no matter how large $z$, it is never optimal to order an infinite amount. Still, it may be that to maximise (11), $T^{*}>>1$.

The tendency of the reference NPV model to arrive at large and unrealistic $T^{*}$ values shows that the model has not sufficiently captured the 'real-world'. Even without having to invoke the uncertainty about demand in the far future, natural constraints on cycle times include warehouse capacities and the fact that a supplier will not be prepared to give unlimited credit. The latter may be modelled by e.g. a maximum credit delay time constraint $z Q / y \leq \bar{L}$. Because (12) is fairly accurate in the range $\alpha T<<1$, Harris' cost function can still be used by introducing these constraints into the model.

We summarise our findings: (1) $p(E O Q, C / C R, z=0) \rightarrow q(E O Q)$ - use Harris' square root solution with (13); (2) $p(E O Q, C / C R, z>0) \rightarrow \neg q(E O Q)$ - to repair the model, use Harris' cost function with (13) in addition to extra constraints from the real-world that will impose limits to $T$.

Example 5. Let $s=£ 100, y=5,000, \alpha=0.2$, and $w=£ 10$. The supplier offers a trade credit in that it is allowed to settle the payment, wyT, no later than 30 days after receival of the batch.

We have two options for modelling this. The first takes $L=30 / 365$ and $z=0$ in (13) and uses Harris' square root solution to find $T^{*} \approx 52$ days, $h \approx £ 1.97$ per unit of product and time, and $C(T *) \approx £ 1,403$ per unit of time. The second approach takes $L=0$, uses two decision variables $z$ and $T$ in (13) in Harris' cost function, adds the constraint $z T \leq 30 / 365$, and finds $T^{*} \approx 52$ days (as it should), $z^{*} \approx 0.58$, but $h \approx-£ 0.325$ per unit of product and time, and $C(T *) \approx £ 529$ per unit of time. Is there a paradox here? Both models represent the same logistics situation, and end up recommending the implementation of the same solution. However, the holding costs in both models are very different. There is only a paradox if one assumes that holding costs receive their meaning from a rigid definition, but not if one adopts NPVEA as here the definition of the (unit) holding cost results from a constructive interpretation found in an equivalence result with a reference NPV model. Different reference models may lead to different local definitions of what the unit holding cost is to be in a valid decision model. When viewed as a construct in order to arrive at optimal decisions, there is indeed nothing strange about e.g. negative holding costs. In the first reference model the PS is based on the fixed delay model as in Rachamadugu and finds $p \rightarrow q$. Holding costs 
cannot become negative - in the approximation (12) the exponential term is not approximated and part of the effect of the trade credit appears also in the marginal profit term. By using the second reference model we have a credit that is a function of cycle time as in Porteus' model, leading to $p^{\prime} \rightarrow \neg q$. However, repairable equivalence is obtained by the consideration of the cost function and an additional constraint that will limit the size that realistic values for cycle times can take. In the approximation (12) the effect is to be absorbed in full in the holding cost term, with the consequence that holding costs can become negative as well. According to NPVEA, both options are equally valid; they lead in the end to the same overall profits (12), save a small linear approximation error.

\subsection{Revenue rewards, multiple parties, and anchor points}

$\mathbf{p}(\mathbf{E O Q}, \mathbf{C} / \mathbf{C I A})$. Take the model $p(E O Q, C)$ and change the PS with the customer, ceteris paribus, as follows. It is agreed that the customer will pay CIA at regular intervals, say with cycle time $T^{\prime}$, for the products delivered afterwards and upto the next payment is due. The AS for the firm is:

$$
\Pi(T)=r y T^{\prime} \sum_{i=0}^{\infty} \alpha e^{-i \alpha T^{\prime}}-(s+w y T) \sum_{i=0}^{\infty} \alpha e^{-i \alpha T},
$$

and approximated:

$$
\bar{\Pi}(T)=(r-w) y-\frac{s}{T}-\alpha \frac{s}{2}-\alpha w \frac{y T}{2}+\alpha r \frac{y T^{\prime}}{2} .
$$

If only $T$ is variable, then $p(E O Q, C / C I A) \rightarrow q(E O Q)$, but if e.g. $y$ or $r$ are variable (too), equivalence is far from certain. Indeed, $p(E O Q, C / C I A)$ contains extra information as given by the last term in (15): the firm enjoys a revenue reward. This has real significance. For example, an increase in $y$, ceteris paribus, increases the firm's profits according to $(r-w) y-\sqrt{2 s \alpha w y}+\alpha r y T^{\prime} / 2$ : marginal profit increases linearly, logistics cost with the square root, and revenue reward linearly. The revenue reward's relative importance increases with $r T^{\prime} / w$.

Example 6. Let $y=1,000, w=£ 50, r=£ 100, s=£ 100, \alpha=0.2$, and $T^{\prime}=0.1$. Then $T^{*}=0.1414,(r-w) y=£ 50,000, C\left(T^{*}\right)=£ 1,414$, and revenue reward $\alpha r \frac{y T^{\prime}}{2}=£ 1,000$. The revenue reward more than compensates for the holding cost of the firm (which is $C\left(T^{*}\right) / 2=£ 707$ ), and represents $2 \%$ of the marginal profit. As no fixed overhead costs have been allocated in this example, the relative importance of both logistics cost and revenue reward to profits may be severly underestimated. 
It is according to the MO of NPVEA valid to introduce a different set of parties with which the firm interacts, or to deviate from the common (implicitly adopted) assumption that the anchor point is located at decision time 0 . The next example illustrates.

Example 7. p(EOQ, 5). Consider a scenario for the EOQ model with five external parties: the customer(s), the supplier, the workforce, a transport company, and an insurance firm. Let $t$ denote the arrival of a batch at the firm. Placement of the order at the supplier occurs at time $t-L_{0}$, at which time the firm incurs an internal order cost $s_{0}$ (independent of the lot-size). The lead-time for the physical transport of the batch from the supplier to the firm is $L_{t}<L_{0}$. The transporter charges the fixed fee $t_{f}$ (independent of the lot-size) and a variable fee $t_{v}$ per product. The fraction $x_{t}$ of the total bill, $t_{f}+t_{v} Q$, is to be paid at time $t-L_{t}$, and the remainder at time $t+L_{t}$. The supplier charges a unit price $w$, and requires $x_{s}$ percent to be paid (per product ordered) as a deposit when placing the order, and the remainder to be settled at time $t+L_{s}+z_{s} Q / y$. A material handling cost per product $g$ is incurred at time $t$. An insurance cost against theft or fire to cover the average inventory level is paid out to an insurance company as an annuity stream of $f$ per product and unit of time. The customers pay $r$, the unit sales price, $L_{r}$ time units after product transfer.

As some payments occur before the arrival of the first batch, the firm has to plan ahead. We therefore introduce an Anchor Point (Beullens and Janssens, 2011) at the arbitrary fixed time $L$ into the future, $L \geq L_{0}$, from which point onwards demand is to be fulfilled. The first batch will thus have to arrive at this time $L$. The annuity stream profit function, discounting all relevant future cash-flows to decision time 0 , is:

$$
\begin{aligned}
\Pi(T)= & {\left[r y e^{-\alpha L_{r}}-f \frac{y T}{2}-\left(s_{0} e^{\alpha L_{0}}+x_{t}\left(t_{f}+t_{v} y T\right) e^{\alpha L_{t}}+\left(1-x_{t}\right)\left(t_{f}+t_{v} y T\right) e^{-\alpha L_{t}}\right.\right.} \\
& \left.\left.+x_{s} w y T e^{\alpha L_{0}}+\left(1-x_{s}\right) w y T e^{-\alpha\left(L_{s}+z_{s} T\right)}+g y T\right) \sum_{i=0}^{\infty} \alpha e^{-i \alpha T}\right] e^{-\alpha L} .
\end{aligned}
$$

We can treat all exponential terms not in function of $T$ as constants and leave them unapproximated. Maclaurin expansion of exponential terms with respect to $\alpha T$ and $\alpha z_{s} T$ in the closed-form expression corresponding to (16), and retaining only the terms linear in $\alpha$, gives the following approximation:

$$
\bar{\Pi}(T)=\left[\left(r e^{\alpha L_{r}}-x_{t} t_{v} e^{\alpha L_{t}}-\left(1-x_{t}\right) t_{v} e^{-\alpha L_{t}}-x_{s} w e^{\alpha L_{0}}\right.\right.
$$




$$
\begin{gathered}
\left.-\left(1-x_{s}\right) w e^{-\alpha L_{s}}-g\right) y-\frac{\left(s_{0} e^{\alpha L_{0}}+x_{t} t_{f} e^{\alpha L_{t}}+\left(1-x_{t}\right) t_{f} e^{-\alpha L_{t}}\right)}{T} \\
-\alpha \frac{\left(s_{0} e^{\alpha L_{0}}+x_{t} t_{f} e^{\alpha L_{t}}+\left(1-x_{t}\right) t_{f} e^{-\alpha L_{t}}\right)}{2}-\alpha\left(x_{t} t_{v} e^{\alpha L_{t}}\right. \\
\left.\left.+\left(1-x_{t}\right) t_{v} e^{-\alpha L_{t}}+x_{s} w e^{\alpha L_{0}}+\left(1-x_{s}\right) w\left(1-2 z_{s}\right) e^{-\alpha L_{s}}+g+\frac{f}{\alpha}\right) \frac{y T}{2}\right] e^{-\alpha L} .
\end{gathered}
$$

Comparing (17) with Harris' cost function gives:

$$
\begin{gathered}
s=s_{0} e^{\alpha L_{0}}+x_{t} t_{f} e^{\alpha L_{t}}+\left(1-x_{t}\right) t_{f} e^{-\alpha L_{t}}>0, \\
h=f+\alpha\left(x_{t} t_{v} e^{\alpha L_{t}}+\left(1-x_{t}\right) t_{v} e^{-\alpha L_{t}}\right. \\
\left.+x_{s} w e^{\alpha L_{0}}+\left(1-x_{s}\right) w\left(1-2 z_{s}\right) e^{-\alpha L_{s}}+g\right) .
\end{gathered}
$$

The right-hand-side of (19) is in general not guaranteed strictly positive; a situation encountered earlier in $p(E O Q, C / C R)$.

To summarise, the equivalence result and the interpretation of the classic model depend on how we build the reference model, in particular what the payment structures are, and what we consider to be decision variables. AS approximations should be thought of as containing in general both capital costs and rewards, and the unit holding cost as a parameter that is unrestricted in sign. When it is non-positive in the EOQ model, the square root solution is inapplicable. Additional information from the real world may become important, such as e.g. capacity constraints or conditions imposed by external parties. We may have a choice when it comes to defining the unit holding cost for describing the same 'real-world' activity and its associated payment structures. An inventory theory that is in first order equivalent to NPV optimisation may need to settle with a definition of the unit holding cost that is to be derived from a constructive interpretation obtained from an equivalence result with an NPV reference model.

\section{The EPQ model}

The third graph in Figure 2 displays the familiar representation of the Economic Production Quantity (EPQ) model (Taft, 1918), a well-known variation of the EOQ model whereby the batch is produced at a finite production rate $R(>y)$, with cost function $C(T)=s / T+h(1-y / R) y T / 2$, for which 
$\left.T^{*}=\sqrt{2 s /[y h(1-y / R)}\right]$, and $C^{*}=\sqrt{2 \operatorname{shy}(1-y / R)}$. Similar to the EOQ, we write that $q(E P Q)$ expresses the applicability of the class of model instances $\{C(T \mid s, y, h, R) \mid s, h, y, R>0, R>y, T>$ $0\}$.

To find an NPV reference model, we provide the PSs between the firm and the outside world consisting of a customer, a labour force, and a third party. Let $r$ be the unit sales price; the PS with the customer is based on an event stream at rate $y$ producing an event payment stream at rate $+r y$. Note that if production starts at the beginning of a cycle, say at $t$, it lasts until $t+y T / R$. Let $w$ be the unit variable production cost; the PS with the labour force involves an event stream at rate $R$ during production, and this gives an event payment stream at rate $-w R$ during that period. Let $s$ be a fixed cost and $f$ a cost per unit of product and unit of time; the PS with the third party consists of the event payment of $-s$ at the start of each production run, and an event payment stream at the rate of $-f$ per unit of product in inventory and unit of time.

$\mathbf{p}(\mathbf{E P Q}, \mathbf{C})$. Adopt C for all PSs; see Figure 2, bottom graph. The AS profit function of the firm is:

$$
\Pi(T)=r y-\left[s+\frac{w R}{\alpha}\left(1-e^{-\alpha \frac{y T}{R}}\right)+F\right] \sum_{i=0}^{\infty} \alpha e^{-i \alpha T},
$$

where:

$$
F=f \int_{0}^{y T / R}(R-y) t e^{-\alpha t} d t+f \int_{y T / R}^{T} y(T-t) e^{-\alpha t} d t
$$

Working out the integrals of $F$, gives the following closed form solution for $F \sum_{i=0}^{\infty} \alpha e^{-i \alpha T}$ :

$$
\frac{f}{\alpha}\left[R \frac{1-e^{-\alpha \frac{y T}{R}}}{1-e^{-\alpha T}}-y\right]
$$

The linearised function, after some algebraic manipulation, is then:

$$
\begin{gathered}
\bar{\Pi}(T)=(r-w) y-\frac{s}{T}-\alpha \frac{s}{2} \\
-(\alpha w+f)\left(1-\frac{y}{R}\right) \frac{y T}{2}-\alpha \frac{f}{6}\left[1-3 \frac{y}{R}+2 \frac{y^{2}}{R^{2}}\right] T \frac{y T}{2} .
\end{gathered}
$$

Assuming $f<<\alpha w$, we can further drop the last term from $(23)$. We hence find $p(E P Q, C) \rightarrow$ $q(E P Q)$. Without loss of insight, take $f=0$ in the reference models that follow.

$\mathbf{p}(\mathbf{E P Q}, \mathbf{C} / \mathbf{C I A})$. Take the model $p(E P Q, C)$ and change the PS with the workforce, ceteris 
paribus, by assuming that $x$ percent of $w$ needs to be paid for at the start of the cycle, while the remainder is still incurred the moment the product has been produced. It is easy to derive that:

$$
\bar{\Pi}(T)=(r-w) y-\frac{s}{T}-\alpha \frac{s}{2}-\alpha\left[x w+(1-x) w\left(1-\frac{y}{R}\right)\right] \frac{y T}{2} .
$$

Thus $p(E P Q, C / C I A, x>0) \rightarrow \neg q(E P Q)$; for $x=1$, Harris' EOQ model should be used, and for $0<x<1$, a blend of the EOQ and EPQ models. We find that $T^{*}(E P Q, C / C I A, x>0) \geq$ $T^{*}(E P Q, C)$, and $C^{*}(E P Q, C / C I A, x>0) \geq C^{*}(E P Q, C)$. The latter is logical, as incurring costs earlier decreases the NPV of this activity for the firm.

$\mathbf{p}(\mathbf{E P Q}, \mathbf{C} / \mathbf{C R})$. Take the model $p(E P Q, C)$ and change the PS with the customer, ceteris paribus, by assuming that the customer is to pay for the lot-size produced upon completing the production run.

The position of the Anchor Point (AP) in this supply chain will now affect the NPV function. Placing the AP at the start of production, which we may assume to be at time 0 , and denoting the model as $p\left(E P Q_{0}, C / C R\right)$, leads to the following AS function:

$$
\Pi_{0}(T)=\left[r y T e^{-\alpha \frac{y T}{R}}-s-\frac{w R}{\alpha}\left(1-e^{-\alpha \frac{y T}{R}}\right)\right] \sum_{i=0}^{\infty} \alpha e^{-i \alpha T},
$$

and approximated:

$$
\bar{\Pi}_{0}(T)=(r-w) y-\frac{s}{T}-\alpha \frac{s}{2}-\alpha\left[w\left(1-\frac{y}{R}\right)-r\left(1-2 \frac{y}{R}\right)\right] \frac{y T}{2} .
$$

From (26) it follows that $h=\alpha\left[w\left(1-\frac{y}{R}\right)-r\left(1-2 \frac{y}{R}\right)\right]^{4}$, and $p\left(E P Q_{0}, C / C R\right) \rightarrow \neg q(E P Q)$. If $R \leq 2 y$, then $+\alpha r(1-2 y / R) y T / 2 \leq 0$ and it represents an opportunity cost, increasing the holding costs of the firm, hence $h>0$ is assured and the square root solution $T^{*}=\sqrt{2 s /(y \alpha[w(1-y / R)-r(1-2 y / R)])}$ applies. If $R>2 y$ then this term represents a revenue reward. In that case, it must be that $r<(R / y-1) w /(R / y-2)$ in order for $h>0$ and the square root solution to apply; else ' $T^{*} \rightarrow \infty$ '. The latter result is, as before in $p(E O Q, C / C R)$, to be interpreted as a sign that additional constraints from the real-world need to be incorporated to limit $T$ to its valid range; or else that the use of higher

\footnotetext{
${ }^{4}$ This is one possible interpretation, in which the effect of $R$ and $r$ are incorporated into the unit holding cost. Another interpretation would split the last term into two terms, one being then the classic holding cost term in $w$, and the second either an additional opportunity cost or reward in $r$.
} 
order models is preferable. Indeed, in this case the linear approximation cannot capture the fact that the optimal cycle time may well be very large, but cannot be infinite. This is intuitively clear as well, since the latter would imply that the firm would have to wait an infinite time before it receives its first revenue cash-flow.

It is also possible to have the AP at another moment relative to the set of events in this activity. For example, one may assume that the AP corresponds to the time when the first payment is due. If the time when the first production run starts is $t$, for example, then the AP would be at time $t+y t / R$. Let this AP be the arbitrary fixed moment in future time $L$ relative to decision time 0 , sufficiently ahead into the future so that $L>y T / R$ for all reasonable scenarios considered. Then the AS of this model $p\left(E P Q_{L}, C / C R\right)$ is:

$$
\Pi_{L}(T)=\left[\left(r y T-\left(s+\frac{w R}{\alpha}\left(1-e^{-\alpha \frac{y T}{R}}\right)\right) e^{\alpha \frac{y T}{R}}\right) \sum_{i=0}^{\infty} \alpha e^{-i \alpha T}\right] e^{-\alpha L}
$$

and approximated:

$$
\bar{\Pi}_{L}(T)=\left[(r-w) y-\frac{s}{T}-\alpha \frac{s}{2}-\alpha\left[w\left(1-\frac{y}{R}\right)-\left(r-w \frac{2 y}{R}\right)\right] \frac{y T}{2}\right] e^{-\alpha L} .
$$

It follows $p\left(E P Q_{L}, C / C R\right) \rightarrow \neg q(E P Q)$, and the last term can be either an opportunity cost or a reward. The behaviour is now very different to the case (26). Rewriting the last term in (28) as:

$$
-\alpha\left[w\left(1+\frac{y}{R}\right)-r\right] \frac{y T}{2},
$$

shows that if $r<w(1+y / R)$, then $T^{*}=\sqrt{2 s / y(w(1+y / R)-r)}$, else ' $T{ }^{*} \rightarrow \infty$ '.

In conclusion, we find that changing the PSs with supplier or customer leads to variations to the classic EPQ inventory model. In some of these variations, as in $p(E P Q, C / C R)$, the unit holding cost is unrestricted in sign, and similar conclusions as reached before in Section 4 apply. The optimal cycle time depends on the anchor point in the supply chain. Its position is likely determined by the practical application, see also Beullens and Janssens (2011). It may arguably make more sense in this example to use (26) instead of (28) in the case that the customer desires the first product at a given time, as this moment will be a function of the optimal cycle time when using (28). 


\section{Lot-sizing in the buyer-supplier supply chain}

Joglekar (1988) developed a model for a buyer-supplier situation in which the unit transfer price $w$ charged between them is explicitly incorporated in an aim to derive the optimal price-discount scheme for the vendor. The buyer faces an EOQ-type problem. He charges the unit sales price $r$ to satisfy demand at a constant rate $y$ per unit of time. His set-up cost is $s_{b}$. The average profit function is:

$$
P_{b}(T)=(r-w) y-\frac{s_{b}}{T}-\alpha_{b} w \frac{y T}{2},
$$

where $\alpha_{b}$ is the buyer's capital cost rate, and $T$ the order cycle time. The supplier produces in batches of equal size $m y T$, where $m \geq 1$ and integer, with production rate $R(>y)$. For initiating production, the set-up cost $s$ is incurred, and $s_{p}$ for delivering a batch $y T$ to the buyer. His average profit function is:

$$
P(m, T)=(w-c) y-\left(\frac{s}{m}+s_{p}\right) \frac{1}{T}-\alpha c\left[(m-1)-(m-2) \frac{y}{R}\right] \frac{y T}{2},
$$

where $\alpha$ is his capital cost rate, and $c$ the unit production cost. See also Figure 3 .

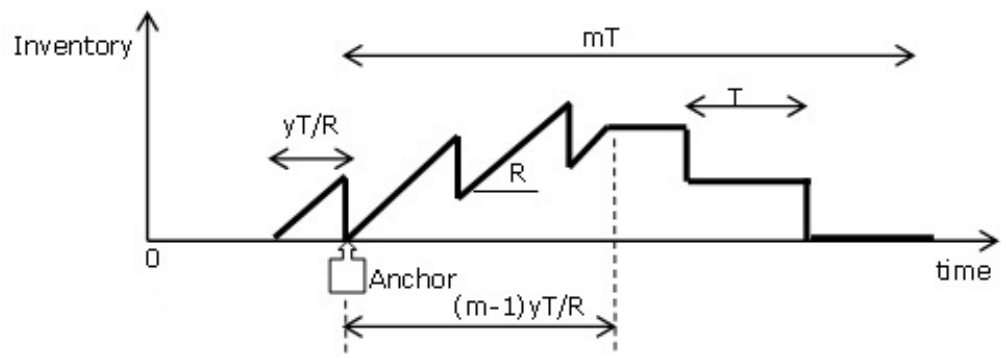

Figure 3: Inventory at the supplier in Joglekar's model (example).

Special cases are: lot-for-lot production (set $m=1$ ), first presented by Banerjee (1986); infinite production rate $(R \rightarrow \infty)$, see Goyal (1988) and also Goyal (1976); and their combination $m=1$ and $R \rightarrow \infty$ as in Monahan (1984). In these models, $\alpha_{b} w$ can be interpreted as the unit holding cost $h_{b}$ for the buyer, and $\alpha c$ as the unit holding cost $h$ for the supplier. The literature on this set of models is extensive, and the search for equivalence hence of great value.

The purpose is to determine the economic order and production quantities, and aspects of Harris' EOQ and Taft's EPQ model are combined. Let $q\left(E O Q ; E P Q^{\prime}\right)$ be used to describe the applicability of the class of models $\left\{\left(P_{b}\left(T \mid r, w, s_{b}, \alpha_{b}, y\right) ; P\left(m, T \mid w, c, y, s, s_{p}, \alpha, R\right)\right) \mid r, w, s_{b}, \alpha_{b}, y, c, s, s_{p}, \alpha>0\right.$, 
$R>y, T>0, m \geq 1$ and integer $\}$.

p(EOQ;EPQ', C). The event time for a set-up is at the start of a cycle; the event times for sales whenever a sales occurs; the event times for production whenever a product has been produced. Note that there are two cycles. The buyer has cycle time $T$, and the supplier cycle time $m T$, and they are nested. In every cycle of the supplier, there is a moment, say $t$, when the first batch of this run is delivered to the buyer. Production of this run, however, starts earlier at time $t-y T / R$, and lasts until $t+(m-1) y T / R$. We include the out-of-pocket cost $f$ per unit of product and time for the supplier, and a similar cost $f_{b}$ for the buyer. We keep using $r$ as unit sales price and $w$ as unit wholesale price. Adopt $\mathrm{C}$ for all PSs.

We again need to be aware of the impact of the AP. We place it at the moment that the first batch is delivered to the buyer, and denote this arbitrary moment in the future as $L$ relative to decision time 0 , sufficiently ahead into the future so that $L>y T / R$ for all reasonable scenarios considered (see also Figure 3). The AS profit function for the buyer is:

$$
\Pi_{b L}(T)=\left[r y-\left(s_{b}+w y T+\int_{0}^{T} f_{b} e^{-\alpha_{b} t} y(T-t) d t\right) \sum_{i=0}^{\infty} \alpha_{b} e^{-i \alpha_{b} T}\right] e^{-\alpha_{b} L}
$$

and for the supplier:

$$
\begin{gathered}
\Pi_{L}(m, T)=\left[-s e^{\alpha \frac{y T}{R}}\left(\sum_{i=0}^{\infty} \alpha e^{-i \alpha m T}\right)+\left(w y T-s_{p}\right)\left(\sum_{i=0}^{\infty} \alpha e^{-i \alpha T}\right)\right. \\
\left.-c R e^{\alpha \frac{y T}{R}}\left(\int_{0}^{y m T / R} e^{-\alpha t} d t\right)\left(\sum_{i=0}^{\infty} \alpha e^{-i \alpha m T}\right)-F\right] e^{-\alpha L},
\end{gathered}
$$

where $F$ measures the impact of the out-of-pocket costs. It can be shown that the linear approximations, retaining only the out-of-pocket costs that are constant terms in $\alpha$, are given by:

$$
\begin{gathered}
\bar{\Pi}_{b L}(T)=(r-w) y-\frac{s_{b}}{T}-\left(\alpha_{b} w+f_{b}\right) \frac{y T}{2} \\
\bar{\Pi}_{L}(m, T)=(w-c) y-\left(\frac{s}{m}+s_{p}\right) \frac{1}{T}-(\alpha c+f)\left[(m-1)-(m-2) \frac{y}{R}\right] \frac{y T}{2} \\
-\alpha \frac{s\left(1+\frac{2 y}{m R}\right)+s_{p}}{2}+\alpha(w-c) \frac{y T}{2} .
\end{gathered}
$$

We find $p\left(E O Q ; E P Q^{\prime}, C\right) \rightarrow \neg q\left(E O Q ; E P Q^{\prime}\right)$, and the two corrections identified are in the 
supplier's profit function. The first correction, $-\alpha\left(s(1+2 y / m R)+s_{p}\right) / 2$, is in contrast to most other NPV models found in the literature, a function of a decision variable, $m$. The second correction, $+\alpha(w-c) y T / 2$, is called the supplier's reward (Beullens and Janssens, 2011; Beullens et al., 2013) and represents the opportunity reward for the supplier due to the fact that larger batch sizes imply that the supplier sees his revenues earlier. While the supplier's reward does not affect the supplier's optimal production quantity when the buyer's order quantity is given, its impact is typically significant in the context of quantity discounts, global optimisation of lot-sizes across the supply chain, and profit sharing, and it is conceptually important in understanding the connection with multi-echelon theory (Clark, 1958; Clark and Scarf, 1960), see Beullens et al. (2013). Note that it is independent of $R$ and $m$, and hence relevant to all special cases of Joglekar's model.

$\mathbf{p}(\mathbf{E O Q} ; \mathbf{E P Q}, \mathbf{C} / \mathbf{C R})$. Take the model $p\left(E O Q ; E P Q^{\prime}, C / C R\right)$ and change the PS with the buyer, ceteris paribus, by having the buyer pay the supplier for a product the moment that the buyer generates a sales. This is known as full consignment. Equivalence analysis shows that the impact on the buyer is that his holding costs reduce to $f_{b} \frac{y T}{2}$, and on the supplier that his supplier's reward becomes the negative value $-\alpha c \frac{y T}{2}$. Hence, in contrast to what is concluded in Huang and Chen (2009) and Hill and Omar (2006), the impact of the change from the conventional PS to full consignment on the supplier's profit function is of size $-\alpha w \frac{y T}{2}$ and not $-\alpha c \frac{y T}{2}$. Note that, under the assumption of symmetry, the cash-flow gain of the buyer must equal the cash-flow loss of the supplier, and hence one must recognise the supplier's reward to arrive at consistent conclusions.

$\mathbf{p}\left(\mathbf{E O Q} ; \mathbf{E P Q}\right.$ ', C/CIA). Take the model $p\left(E O Q ; E P Q^{\prime}, C\right)$, simplify by setting $f=0$, and change the PS of the production process for the supplier, ceteris paribus, by requiring payment of the complete cost cmyT for a run to be incurred at the start of production. The impact for the supplier is a change of the term in his AS function related to production, which now becomes:

$$
-c m y T e^{\alpha \frac{y T}{R}} \sum_{i=0}^{\infty} \alpha e^{-i \alpha m T}
$$

leading to a holding cost in the linear approximation given by:

$$
-\alpha c\left[(m-1)+2 \frac{y}{R}\right] \frac{y T}{2},
$$

where $(m-1)$ arises due to the fact that $-\alpha c y T / 2$ is to be allocated to the supplier's reward, which 
is still given by $\alpha(w-c) y T / 2$. The optimal value for $m$ is now only dependent on $R$ via the set-up cost $s$, see (35). A larger value for $R$ leads to holding cost savings for the firm, whereas under $\mathrm{C}$ it would typically be advantageous to have $R \rightarrow y$.

$\mathbf{p}(\mathbf{E O Q} ; \mathbf{E P Q}, \mathbf{C} / \mathbf{C R} 2)$. Take the model $p\left(E O Q ; E P Q^{\prime}, C\right)$, simplify by setting $f=0$, and change the PS of the production process for the supplier, ceteris paribus, by requiring payment of the cost $c y T$ when the supplier ships to the buyer. This would be the analogue of the full consignment approach, but now between the supplier and his manufacturing process. The change to the production cost in the supplier's AS is:

$$
-c y T \sum_{i=0}^{\infty} \alpha e^{-i \alpha T},
$$

leading, in the linear approximation, to a zero holding cost and the supplier's reward $\alpha(w-c) y T / 2$. The production rate $R$ has a limited effect on the profits of the firm, and ' $m^{*} \rightarrow \infty$ ' (as always, then some additional constraint must be imposed for the linear approximation to be applicable).

p(EOQ;EPQ', C/CR3). Consider a variation to the previous case in which the total production cost $c m y T$ is paid only when the supplier completes a production run. The change to the production cost in the supplier's AS is:

$$
-c m y T e^{-\alpha(m-1) \frac{y T}{R}} \sum_{i=0}^{\infty} \alpha e^{-i \alpha m T},
$$

leading, in the linear approximation, to a holding cost:

$$
-\alpha c(m-1)\left(1-2 \frac{y}{R}\right) \frac{y T}{2},
$$

and a supplier's reward $\alpha(w-c) y T / 2$. Hence, holding costs for $m>1$ can become non-positive when $R \leq 2 y$, in which case ' $m^{*} \rightarrow \infty$ '.

The inclusion of out-of-pocket costs will add an element of the original model under $\mathrm{C}$ to the holding costs, but not significantly affect the main findings. The derivation of the functions can be repeated for the case of the AP at the start of production. One will then find that both the buyer's and supplier's holding costs deviate significantly from the classic formulation, see also Beullens and Janssens (2011) for examples of the special cases that either $m=1$ or $R \rightarrow \infty$. While in all cases considered above we find non-equivalence, we have also stated at the end of Section 2.1 that the quest for equivalence is never completed since $p \neq \mathcal{P}$. Beullens et al. (2013) prove that the set of functions 
(30) and (31), viewed from within a set of axiomatic assumptions ${ }^{5}$, cannot lead to equivalence for any payment structure through which the buyer will directly pay its supplier, and hence the result:

$$
\forall p \in P^{\prime}: p \rightarrow \neg q
$$

where $P^{\prime}$ is the universe of all possible PSs between buyer and vendor that satisfy their axiomatic framework. While this result seems to carry some weight, $P^{\prime} \neq P$, and there are still an infinite number of other reference NPV models outside $P^{\prime}$ that may lead to valid interpretations. Indeed, Beullens et al. (2013) provide one such non-trivial valid interpretation.

\section{Changing the physical flows, ceteris paribus}

So far, we have adopted the physical flows as given by the classic model, and have illustrated the impact of changing payment structures. In this section the point is made that the physical flows have no impact on the NPV reference model as long as the cash-flow functions remain the same, i.e. if payment structures (and the events on which they are based) remain unaltered. From this follows that an inventory theory based on integration (1) will in general fail the test of equivalence.

As a first example, take the EOQ model, but assume that as soon as a batch arrives, it is immediately delivered in full to the customer, while keeping the PS as if we had a normal EOQ model under C. (This example is based on Porteus (1985).) Even if $f \neq 0$, the inventory and thus the out-of-pocket costs are zero, and since the PSs have not changed, we can use the results for $p(E O Q, C)$ by setting $f=0$. Hence Harris' model can still be used. The use of (1) would no longer produce an equivalent result as we would obtain:

$$
\frac{1}{T} \int_{0}^{T} h(t) I(t) d t=\frac{1}{T} \int_{0}^{T}(\alpha w+f) 0 d t=0
$$

giving $C(T)=s / T$, which is minimised for $T^{*} \rightarrow \infty$. This cannot be right, since we know from the equivalence analysis that the equivalent function is $C(T)=s / T+\alpha w y T / 2$ and hence the policy is still Harris' EOQ formula $T^{*}=\sqrt{2 s / y \alpha w}$ for $f=0$.

Consider, as a second example, the case as in the first example, but now assume that from the

\footnotetext{
${ }^{5}$ Which are in this case: (1) the Assumption of Symmetry (see Section 3.1) for the cash-flow between buyer and vendor, and (2) the assumption that $\alpha_{b}=\alpha>0$.
} 
moment a batch arrives, the products are delivered to the customer at the rate $2 y$, ceteris paribus. We can use the function obtained from $p(E O Q, C)$, and only need to recalculate the impact of $f$. So, in case that $f=0$, the physical delivery pattern to the customer can deviate arbitrarily large from the normal assumptions of the EOQ model. Using classic integration, we would obtain:

$$
\frac{1}{T} \int_{0}^{T} h(t) I(t) d t=\frac{1}{T} \int_{0}^{T / 2}(\alpha w+f) y(T-2 t) d t=h y T / 4
$$

and hence $C(T)=s / T+h y T / 4$ is minimised for $T^{*}=\sqrt{4 s / y h}$. This cannot be right, since we know from the equivalence analysis that the equivalent cost function is $C(T)=s / T+\alpha w y T / 2+f y T / 4$, giving $T^{*}=\sqrt{2 s / y(\alpha w+2 f)}$.

The usefulness of (1), i.e. classic integration, would therefore be limited in situations where the physical flows no longer follow classic modeling assumptions. In this sense, an inventory theory that is to be a first order theory on NPV optimisation would not only have to derive its definition of the unit holding cost from a constructive interpretation obtained from an equivalence result with a reference NPV model (see Section 4), but also do this as to derive its method of calculating the holding cost. Using a reference model in the (initial) investigation will in addition help with the identification of any opportunity rewards or other phenomena that might be of importance in setting up an appropriate decision model.

Having made this point, we do think it less likely that when confronted with the above physical flows, which clearly differ from classic patterns, one would apply (1). An understanding of the classic interpretation (2) of $h$, in which the investment $v$ conventionally occurs when an order arrives, should trigger warnings about the straightforward use of (1) in such cases. Using a reference NPV model offers the advantage of not having to rely on intuition, but having a mathematical process that will guide us towards the correct model.

We have just illustrated that when changing physical flows in such a way that they leave cashflows intact for a firm, the result is that this keeps the inventory model in the profit function for this firm being exactly the same. We can hence, for example, also re-interpret $p\left(E O Q ; E P Q^{\prime}, C / C R 3\right)$ as describing the profit function of the 'supplier' when production and delivery to the customer are outsourced to a third party, who charges the 'supplier' a set-up cost $s$ at the start of the run, a delivery charge $s_{p}$ whenever a batch is delivered to the customer, and cmyT upon completion of a production run. Note that (40) then still correctly describes a 'holding cost' (or reward, if negative) 
for the supplier for products he does not carry himself! While it may be that ' $m^{*} \rightarrow \infty$ ' for the supplier, the third party producer will disagree (as his production costs may be incurred at other times) and aim to propose the value of $m$ that would optimise his own process, described by e.g. a variation to $p\left(E O Q ; E P Q^{\prime}, C\right)$. The application of NPVEA, or NPV modelling in general, offers various benefits for the study of supply chains of independent firms that are yet to be exploited in research.

\section{Conclusions and further research}

Classic inventory theory has its roots in an analytical modelling approach which has often been shown to be remarkably accurate and of practical value in a wide range of applications. To help its further development into a theory that is compatible in at least first order with the Net Present Value paradigm, we have formalised an approach that has been in the making at least since 1963, illustrated how this can deal with various payment structures to produce a wide range of possible reference models, and how this leads to different interpretations of, or variations to, classic inventory models.

The examples presented illustrate that the method, which we call NPVEA, is a useful modelling and theory development tool in that it can indicate which parts of an existing inventory theory are worth preserving and where perhaps some modifications are in order. The most important idea is not the linear approximation, but is due to the information gap which gives rise to an interpretation problem. Therefore, we cannot rely on the parameters that have become of integral importance in classic inventory theory, when formulating the reference model. The most important practical aspect is thus to start from a different view of the world in which the inventory model resides - a description in terms of a collection of firms that exchange cash-flows with each other in order to execute the activity, where the label 'firms' is to be interpreted very broadly (it can also include employees, consultancy firms, financial institutions, etc). As there are many plausible NPV reference models, there are many plausible valid interpretations of a classic model and its underlying theory. Refinements to this theory can be uncovered, including the need to consider revenue rewards and negative holding costs, as has been illustrated in this paper.

The further application of NPVEA must be useful in the context of examining the ubiquitous but illustrious unit backorder cost and unit lost sales cost in inventory models in which backorders or 
lost sales (are allowed to) occur. The method is not restricted to inventory models, but in principle applicable to any model in which monetary flows over time are of importance but in which the construction of cash-flow functions was not the starting point of model development.

\section{References}

Banerjee, A. 1986. On 'a quantity discount pricing model to increase vendor profits'. Management Science 32(11) 1513-1517.

Beullens, P., G.K. Janssens. 2011. Holding costs under push or pull conditions - the impact of the anchor point. European Journal of Operational Research 215 115-125.

Beullens, P., G.K. Janssens, L.N. Van Wassenhove. 2013. The capital costs and rewards of inventories in supply chains of independent firms. Working paper, University of Southampton, Submitted 34p.

Çorbacioğlu, U., E.A. van der Laan. 2007. Setting the holding cost rates in a two-product system with remanufacturing. International Journal of Production Economics 109 185-194.

Chung, K.J., J.J. Liao. 2009. The optimal ordering policy of the eoq model under trade credit depending on the order quantity from the dcf approach. European Journal of Operational Research $196563-568$.

Clark, A.J. 1958. A dynamic, single-item, multi-echelon inventory model. RM-2297, Santa Monica, California, The RAND Corporation December.

Clark, A.J., H. Scarf. 1960. Optimal policies for a multi-echelon inventory problem. Management Science 6(4) 475-490.

Deutsch, D. 2011. The Beginning of Infinity. Explanations that Transform the World. Viking Penguin, Penguin Group (USA).

Disney, S.M., R.D.H. Warburton. 2012. On the lambert w function: Economic order quantity applications and pedagogical considerations. International Journal of Production Economics 140(2) 756-764. 
Goyal, S.K. 1976. An integrated inventory model for a single supplier - single customer problem. Int. J. of Production Research 15(1) 107-111.

Goyal, S.K. 1988. A joint economic-lot-size model for purchaser and vendor: A comment. Decision Sciences 19 236-241.

Grubbström, R.W. 1967. On the application of the laplace transform to certain economic problems. Management Science 13(7) 558-567.

Grubbström, R.W. 1980. A principle for determining the correct capital costs of work-in-progress and inventory. International Journal of Production Research 18(2) 259-271.

Grubbström, R.W. 2007. Transform methodology applied to some inventory problems. Zeitschrift für Betriebswirtshaft 77(H.3) 297-324.

Grubbström, R.W. 2013. Dynamic lotsizing with a finite production rate. International Journal of Production Economics http://dx.doi.org/10.1016/j/jipe.2012.12.009i.

Gurnani, C. 1983. Economic analysis of inventory systems. Int. J. of Production Research 21(2) $261-277$.

Hadley, G. 1964. A comparison of order quantities computed using the average annual cost and the discounted cost. Management Science 10(3) 472-476.

Hadley, G., T. Whitin. 1963. Analysis of Inventory Systems. Prentice-Hall, Englewood Cliffs, NY.

Haneveld, W.K.K., R.H. Teunter. 1998. Effects of discounting and demand rate variability on the eoq. International Journal of Production Economics 54 173-192.

Harris, F.W. 1913. How many parts to make at once. Factory, The Magazine of Management 10(2) $135-136,152$.

Hill, R.M., M. Omar. 2006. Another look at the single-vendor single-buyer integrated productioninventory problem. International Journal of Production Research 44(4) 791-800.

Huang, Q., J. Chen. 2009. A note on: Modelling an industrial strategy for inventory management in supply chains: the 'consignment stock' case. International Journal of Production Research 47(22) 6469-6475. 
Joglekar, P.N. 1988. Comments on 'a quantity discount pricing model to increase vendor profits'. Management Science 34(11) 1391-1398.

Monahan, J.P. 1984. A quantity discount pricing model to increase vendor profits. Management Science 30(6) 720-726.

Porteus, E.L. 1985. Undiscounted approximations of discounted regenerative models. Operations Research Letters 3(6) 293-300.

Rachamadugu, R. 1989. Effect of delayed payments (trade credit) on order quantities. Journal of the Operational Research Society 40(9) 805-813.

Silver, E.A., D.F. Pyke, R. Peterson. 1998. Inventory Management and Production Planning and Scheduling, 3th. ed.. John-Wiley and Sons, New York.

Taft, E.W. 1918. The most economical production lot. Iron Age 101 1410-1412.

Teng, J.T., C.T. Chang, M.S. Chern, Y.L. Chan. 2007. Retailer's optimal ordering policies with trade credit financing. International Journal of Systems Science 38(3) 269-278.

Teunter, R., E. van der Laan. 2002. On the non-optimality of the average cost approach for inventory models with remanufacturing. International Journal of Production Economics 79 67-73.

Teunter, R.H., E. van der Laan, K. Inderfurth. 2000. How to set the holding cost rates in average cost inventory models with reveres logistics? Omega 28 409-415.

Van der Laan, E., R. Teunter. 2002. Average costs versus net present value: a comparison for multisource inventory models. A. Klose, M.G. Speranza, L.N. Van Wassenhove, eds., Quantitative Approaches to Distribution Logistics and Supply Chain Management. Springer-Verlag, 359-378.

Xu, S.X., Q. Lu, Z. Li. 2012. Optimal modular production strategies under market uncertainty: A real options perspective. International Journal of Production Economics 139(1) 266-274. 